\title{
Using single sex-specific high-sensitivity cardiac troponin cut-off values for ruling out myocardial infarction - Are we there yet?
}

\author{
Venkatesh Thiruganasambandamoorthy, MBBS, MSc ${ }^{*} \neq$
}

\section{INTRODUCTION}

Chest pain is the second most common emergency department (ED) presenting complaint. ${ }^{1}$ Chest pain constitutes approximately $5 \%$ of all $\mathrm{ED}$ visits and, in the year 2012, an estimate suggests that there were approximately 600,000 ED visits for chest pain across Canada. ${ }^{2}$ Given that chest pain is very common in the $\mathrm{ED}$, improved efficiency in ED management of patients with suspected myocardial infarction (MI) is a key issue in alleviating ED crowding. Yet this improved efficiency should not compromise safety due to a false-negative diagnosis.

In acute care settings, women in comparison with men present with atypical symptoms, have fewer electrocardiogram abnormalities, and have lower cardiac troponin values for any extent of underlying coronary artery disease. ${ }^{3,4}$ Such differences in troponin values are extremely variable based on the type of assay with large differences with some assay types and negligible with others. ${ }^{5}$ Studies have reported that sex-specific cut-off values for highsensitivity cardiac troponin (hs-cTn) assays improve diagnosis and classification performance of MI, particularly in women, leading to professional society guidelines recommending their use. ${ }^{3,6,7}$ While studies have previously focused on improving ruling-in MI with sexspecific cut-offs, in this issue, McRae and colleagues explore the concept of ruling-out $\mathrm{MI}$ with a single Roche Elecsys ${ }^{\circledR}$ high-sensitivity cardiac troponin $\mathrm{T}$ (hs-c $\left.\mathrm{Tn} \mathrm{T}\right)$, using several cut-off levels relative to the limit of detection $(<5 \mathrm{ng} / \mathrm{L})$ and the Food and Drug Administration- approved limit of quantification $(<6 \mathrm{ng} / \mathrm{L}){ }^{8}$ The 99 th percentile reference limit for this assay is $14 \mathrm{ng} / \mathrm{L}$. The study found that sex-specific cut-offs may improve the classification performance and could lead to rule out MI among more patients on ED arrival. The authors were explicit in their discussion that their study was purely exploratory, and future research is needed in this area.

A few points merit discussions on this topic. The 99th percentile reference limit for a normal population is currently being used to define acute MI. $^{7}$ However, these 99th percentile values are dependent on the population selected and the definition for heathy that is used in these studies. ${ }^{5}$ The study by McRae and colleagues introduces the concept of using sex-specific hs-cTnT cut-off values well below the 99th percentile normal population reference limit, particularly when using a single measurement within 60 minutes of ED arrival for ruling out MI. Hence, moving away from the 99th percentile reference cut-off and using specific values for each assay type that is confirmed in the target population is a step in the right direction for an improved diagnosis of MI. As detailed in the counterpoint argument by Giannitsis, the adoption of ruling in MI with sex-specific cut off values in previous studies has shown very little clinical benefit. ${ }^{9}$ The use of reclassification should be with caution due to inherent confirmation bias, as the troponin values are incorporated in the adjudication of MI outcome in these studies. Future studies are needed to confirm that sex-specific cut-offs improve the diagnosis of MI and specifically the impact on clinically

From the Departments of *Emergency Medicine and TSchool of Epidemiology and Public Health Community Medicine, University of Ottawa, Ottawa, ON; and the ¥Ottawa Hospital Research Institute, The Ottawa Hospital, Ottawa, ON.

Correspondence to: Dr. Venkatesh Thiruganasambandamoorthy, Clinical Epidemiology Unit, The Ottawa Hospital, 1053 Carling Avenue, 6th Floor, Rm F650 Ottawa, Ontario K1Y 4E9; Email: vthirug@ohri.ca

(c) Canadian Association of Emergency Physicians 
important hard outcomes such as mortality, and revascularization procedures should be documented. Additionally, such new sex-specific cut-offs identified must be beyond the range of assay variability for adoption into clinical practice, unlike in the published study.

In summary, further large-scale studies that confirm sex-specific cut-offs beyond assay variability and clinical benefit are needed for the incorporation into day-to-day practice. Such sex-specific cut-offs will unfortunately have to be developed for each assay type in the intended target population.

Keywords: High-sensitivity troponin, myocardial infarction, sexspecific cut-off

Competing interests: None declared.

\section{REFERENCES}

1. Rui PK, Kang K. National Hospital Ambulatory Medical Care Survey: Emergency department summary tables. Atlanta, GA, USA: National Center for Health Statistics; 2015.
2. Scheuermeyer FX, Wong H, Yu E, et al. Development and validation of a prediction rule for early discharge of low-risk emergency department patients with potential ischemic chest pain. C7EM 2014;16:106-19.

3. Slagman A, Searle J, Vollert JO, et al. Sex differences of troponin test performance in chest pain patients. Int 7 Cardiol 2015;187:246-51.

4. Laufer EM, Mingels AM, Winkens MH, et al. The extent of coronary atherosclerosis is associated with increasing circulating levels of high sensitive cardiac troponin T. Arterioscler Thromb Vasc Biol 2010;30:1269-75.

5. Apple FS, Ler R, Murakami MM. Determination of 19 cardiac troponin I and T assay 99th percentile values from a common presumably healthy population. Clin Chem 2012;58:1574-81.

6. Shah AS, Griffiths M, Lee KK, et al. High sensitivity cardiac troponin and the underdiagnosis of myocardial infarction in women: prospective cohort study. BMF 2015;350:g7873.

7. Thygesen K, Alpert JS, Jaffe AS, et al. Third universal definition of myocardial infarction. Circulation 2012;126:2020-35.

8. McRae A, Graham M, Abedin T, et al. Sex-specific, highsensitivity cardiac troponin T cut-off concentrations for ruling out acute myocardial infarction with a single measurement. CFEM 2019;21:26-33.

9. Giannitsis E. Counterpoint: potential concerns regarding the use of sex-specific cutpoints for high-sensitivity troponin assays. Clin Chem 2017;63:264-6. 